\title{
Observed changes in dry-season water availability attributed to human-induced climate change
}

\author{
Ryan S. Padrón ${ }^{1 凶}$, Lukas Gudmundsson $\mathbb{1}^{1}{ }^{1}$, Bertrand Decharme $\mathbb{1}^{2}$, Agnès Ducharne $\mathbb{1}^{3}$, \\ David M. Lawrence $\mathbb{B}^{4}{ }^{4}$, Jiafu Mao ${ }^{(1)}{ }^{5}$, Daniele Peano ${ }^{6}{ }^{6}$, Gerhard Krinner ${ }^{7}{ }^{7}$, Hyungjun Kim ${ }^{8}{ }^{8}$ and \\ Sonia I. Seneviratne ${ }^{1} 1$
}

\begin{abstract}
Human-induced climate change impacts the hydrological cycle and thus the availability of water resources. However, previous assessments of observed warming-induced changes in dryness have not excluded natural climate variability and show conflicting results due to uncertainties in our understanding of the response of evapotranspiration. Here we employ data-driven and land-surface models to produce observation-based global reconstructions of water availability from 1902 to 2014, a period during which our planet experienced a global warming of approximately $1^{\circ} \mathrm{C}$. Our analysis reveals a spatial pattern of changes in average water availability during the driest month of the year over the past three decades compared with the first half of the twentieth century, with some regions experiencing increased and some decreased water availability. The global pattern is consistent with climate model estimates that account for anthropogenic effects, and it is not expected from natural climate variability, supporting human-induced climate change as the cause. There is regional evidence of drier dry seasons predominantly in extratropical latitudes and including Europe, western North America, northern Asia, southern South America, Australia and eastern Africa. We also find that the intensification of the dry season is generally a consequence of increasing evapotranspiration rather than decreasing precipitation.
\end{abstract}

$\mathrm{C}$ hanges in the hydrological cycle with widespread consequences for humans and ecosystems are expected across the world under global warming ${ }^{1-4}$. Water availability on land, determined by precipitation $(P)$ minus evapotranspiration $(\mathrm{ET})^{5-8}$, represents a major concern in this context. Identifying and attributing changes in water availability due to historic human-induced climate change has been hindered by length and quality of observational records ${ }^{1}$, natural climate variability ${ }^{8,9}$ and additional effects from aerosol emissions ${ }^{10}$. Nevertheless, there is growing evidence of human influence on observed $P$ changes ${ }^{11,12}$, ET changes from hydrological models ${ }^{13}$, trends in continental-scale river flow ${ }^{14}$, trends in drought severity ${ }^{10}$ and trends in global root zone soil moisture from reanalysis and climate models ${ }^{15}$.

There are large uncertainties concerning both drought changes in the recent past $^{1}$ and future water availability projections ${ }^{6}$. The ET response to warming is a key component of these uncertainties. Earlier drought assessments with the Palmer Drought Severity Index ${ }^{16}$, a metric that relies on temperature-based potential ET, pointed to a substantial increase in aridity since the $1970 \mathrm{~s}^{17,18}$. However, this was shown to be overestimated by using a modified version of the index that also accounts for available energy, air humidity and wind speed when computing potential $\mathrm{ET}^{19}$. More importantly, climate models indicate that actual ET does not follow the increasing rate of potential ET under warming induced by an increasing concentration of atmospheric $\mathrm{CO}_{2}^{5,20,21}$. In addition, a simplified expectation of enhanced patterns of $P$-ET under global warming has been suggested ${ }^{22}$ but was shown not to apply for annual averages over land ${ }^{6,23,24}$. There are also reports of an observed increase in the annual range between wet and dry season P over land, although mainly due to wetter wet seasons, ${ }^{25}$ as well as projected decreases in dry season $\mathrm{P}$ over the Amazon ${ }^{26}$ and in dry season $P$-ET over most of the Northern Hemisphere, ${ }^{7,8}$.

Here we analyse how dry-season water availability, represented by minimum monthly $P$-ET, has changed over the past century using new observation-based data. On the one hand, we employ recently published datasets of global runoff $(R)^{27}$ and terrestrial water storage fluctuations $(\Delta \text { TWS })^{28}$ from 1902 to 2014 to obtain water availability estimates according to the water balance $P-\mathrm{ET}=R+\Delta$ TWS. The datasets for both $R$ and $\Delta$ TWS are reconstructions from statistical data-driven models (DDM) calibrated with observations, which perform well compared with state-of-the-art hydrological models ${ }^{27,28}$. Near-surface air temperature and $P$ data are used as explanatory variables for both reconstructions. On the other hand, we also employ the mean $P$-ET of multiple land-surface model (LSM) simulations that are driven with observational atmospheric data $^{29}$. The atmospheric forcing data used for both the DDM and LSM reconstructions are from the Global Soil Wetness Project Phase 3 (GSWP3) ${ }^{30}$. Although these reconstructions are not exempt from caveats such as not accounting for land-use changes (DDM), local land-atmosphere feedbacks (LSM) and groundwater withdrawal (DDM and LSM), they are complementary and increase confidence in our analysis.

\footnotetext{
IInstitute for Atmospheric and Climate Science, Department of Environmental Systems Science, ETH Zurich, Zurich, Switzerland. ${ }^{2} \mathrm{CNRM}$, Centre National de Recherches Météorologiques, Université de Toulouse, Météo-France, CNRS, Toulouse, France. ${ }^{3}$ Laboratory METIS (Milieux environnementaux, transferts et interaction dans les hydrosystèmes et les sols, Metis), Sorbonne Université, CNRS, EPHE, IPSL, Paris, France. ${ }^{4}$ Climate and Global Dynamics Laboratory, National Center for Atmospheric Research, Boulder, CO, USA. ${ }^{5}$ Environmental Sciences Division and Climate Change Science Institute, Oak Ridge National Laboratory, Oak Ridge, TN, USA. ${ }^{6}$ Fondazione Centro euro-Mediterraneo sui Cambiamenti Climatici, CMCC, Bologna, Italy. ${ }^{7}$ Institut des Géosciences de l'Environnement (IGE), CNRS, Université Grenoble Alpes, Grenoble, France. ${ }^{8}$ Institute of Industrial Science, The University of Tokyo, Tokyo, Japan.凶e-mail: ryan.padron@env.ethz.ch
} 
Changes in dry-season water availability (in $\mathrm{mm} \mathrm{month}^{-1}$ ) are computed as:

$$
\Delta(P-\mathrm{ET})=(P-\mathrm{ET})_{\text {pres }}-(P-\mathrm{ET})_{\text {past }}
$$

where $(P-\mathrm{ET})_{\text {pres }}$ is the average of minimum monthly $P$-ET from the recent period $1985-2014$ and $(P-\mathrm{ET})_{\text {past }}$ is the average from the reference period 1902-1950. We omit the period 1951-1984, during which anthropogenic aerosol emissions had the highest confounding effect on the response of climate to greenhouse gas emissions $^{10,31}$. In the tropics, we consider minimum $P$-ET from all months of the year, whereas in extratropical latitudes $\left(>23.5^{\circ}\right)$, we focus only on the warmer months: May-September in the Northern Hemisphere and November-March in the Southern Hemisphere.

\section{Pattern of change in dry-season water availability}

Changes in minimum monthly P-ET since the early 1900s are shown throughout the world on the basis of DDM (Fig. 1a) and LSM reconstructions (Fig. 1b). There is an agreement in the sign of the changes between these reconstructions over 59\% of all land grid cells, excluding Antarctica, Greenland and deserts with annual $P$ below $100 \mathrm{~mm}$ (see Extended Data Fig. 1). The DDM (LSM) data indicate a predominant decrease in dry-season water availability over $57 \%(56 \%)$ of the land area, whereas there is increased water availability in the remaining $43 \%$ (44\%) (see also Extended Data Fig. 2). In regions with drying dry seasons, there is a median decrease of $2.1 \mathrm{~mm} \mathrm{month}^{-1}$ since the first half of the twentieth century for the DDM and $2.8 \mathrm{~mm} \mathrm{month}^{-1}$ for the LSM, whereas in regions with wetter dry seasons, the median increase is $1.8 \mathrm{~mm} \mathrm{month}^{-1}$ for the DDM and $2.7 \mathrm{~mm} \mathrm{month}^{-1}$ for the LSM. In addition, note that there is little sensitivity of the observed patterns of change in dry-season water availability to the definition of the reference period (Extended Data Fig. 3). Different stochastic realizations of the $\Delta$ TWS reconstruction (there are no stochastic realizations of the $R$ reconstruction), as well as reconstructions from individual LSMs, also show a general agreement that supports the pattern of $\Delta(P-\mathrm{ET})$ (Extended Data Figs. 4 and 5). Finally, results are generally consistent when analysing minimum 3-monthly $P$-ET, as opposed to monthly (Extended Data Fig. 6).

Both observation-based reconstructions (DDM and LSM) indicate a pattern of enhanced dry seasons at extratropical latitudes. Regions affected by an intensification of the dry season include Europe, western North America, northern Asia, southern South America, Australia, northern Andes and eastern Africa. Approximately three-fourths of the land area in these regions shows reduced water availability during the dry season for at least one of the reconstructions. However, dry-season water availability has also increased elsewhere; for example, in inland China, southeastern Asia and the Sahel.

Climate change signal detected in observed change pattern The multimodel mean of 36 coupled climate model simulations (Extended Data Table 1) with historical radiative forcing also suggests a decrease in dry-season water availability in many regions where this was observed (Fig. 1c). Conversely, simulations that exclude human influence while taking into account only historical natural radiative forcing do not capture the observed changes (Fig. 1d). Some differences are expected between the multimodel mean and observations; for example, the spatially smoother and weaker changes from the multimodel mean arise from averaging over several different possible evolutions of the climate system, whereas the reconstructions correspond to the single observed evolution of the system (see Extended Data Fig. 7 for agreement in the sign of $\Delta(P-E T)$ between the individual climate models). In addition, higher observational and model uncertainties probably contribute to the disagreement in the Amazon and central Africa. Potential
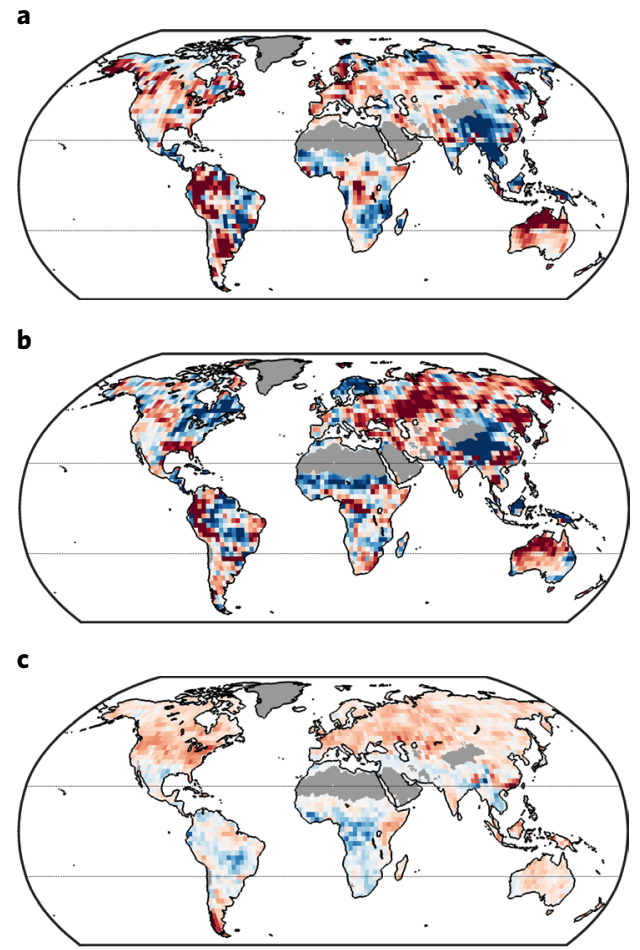

d

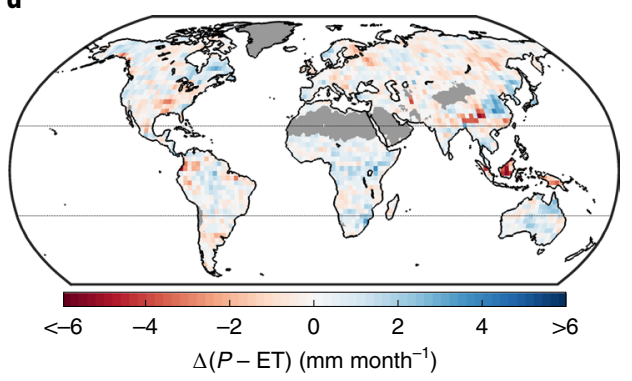

Fig. 1 | Patterns of change in dry-season water availability from observation-based reconstructions and climate models. a, DDM reconstruction. $\mathbf{b}$, LSM reconstruction. $\mathbf{c}, \mathbf{d}$, Simulated changes based on multimodel mean of coupled climate models with historical radiative forcing that includes human-induced emissions (c) and with only natural historical radiative forcing (no human-induced emissions) (d). For $\mathbf{d}$ the latter period ends in 2005 because no further data are available. Grey lines indicate tropical boundaries at $23.5^{\circ} \mathrm{S}$ and $23.5^{\circ} \mathrm{N}$. Antarctica, Greenland and desert regions with annual $P$ below $100 \mathrm{~mm}$ are masked in grey.

differences also may arise from the representation of land use changes. Nonetheless, climate simulations point to human-induced climate change as the underlying reason for the observed pattern of change in dry-season water availability.

To formally assess the influence of anthropogenic climate change on observed average minimum monthly $P$-ET, we formulate the null hypothesis that there is no difference between the pattern of observed changes in water availability and what is expected from natural climate variability. To test this, we first approximate natural climate variability by computing hundreds of estimates of changes in dry-season water availability from climate model simulations forced with pre-industrial conditions $\left(\Delta(P-\mathrm{ET})_{\mathrm{mdl}, \mathrm{pi}}\right)$, while using equivalent $113-y r$ periods as for the observations. Then we obtain the spatial pattern Spearman correlation of each $\Delta(P-\mathrm{ET})_{\mathrm{mdl}, \mathrm{pi}}$ estimate with the multimodel mean change during the same historical period as for the observations $\left(\Delta(P-\mathrm{ET})_{\mathrm{mdl}, \text { hist }}\right)$. The resulting 

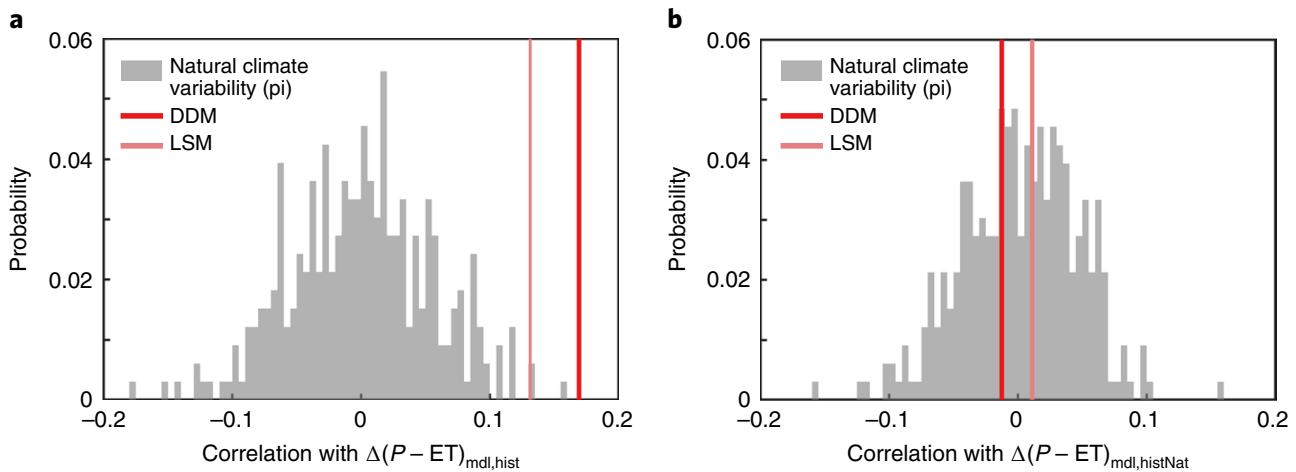

Fig. 2 | Pattern correlations of changes in dry-season water availability between simulations with and without anthropogenic climate change and observation-based reconstructions. a, Spatial pattern Spearman correlation between the multimodel mean from historical simulations with human-induced plus natural radiative forcing and observed changes (that is, correlation of $\Delta(P-E T)_{\text {mdl,hist }}$ and $\Delta(P-E T)_{\text {obs }}$ ) compared with an empirical probability density function of correlations expected from natural climate variability (that is, correlations of $\Delta(P-\mathrm{ET})_{\text {mdl,hist }}$ and 320 estimates of $\left.\Delta(P-E T)_{\text {mdlpi }}\right)$. b. Same as a but for the multimodel mean from historical simulations with natural radiative forcing only, that is, $\Delta(P-E T)_{\text {mdllhistnat }}$ instead of $\Delta(P-E T)_{\text {mdl,hist }}$. Antarctica, Greenland and desert regions with annual $P$ below $100 \mathrm{~mm}$ are omitted when computing the spatial correlations.

distribution gives the range of spatial correlations with the simulated historical estimate that can be expected from natural climate variability alone. Finally, if the correlations of $\Delta(P-E T)_{\text {mdl,hist }}$ with the observed change $\Delta(P-\mathrm{ET})_{\mathrm{obs}}$ given by the DDM and LSM reconstructions are greater than what is expected from natural variability, then we conclude that a response to anthropogenic climate change is detectable in the observed pattern (see Methods).

Our results indicate an extremely likely contribution of human-induced emissions to the observed pattern of changes in dry-season water availability (Fig. 2a), since there is a $99 \%$ probability that the spatial Spearman correlation between $\Delta(P-\mathrm{ET})_{\text {mdl,his }}$ and $\Delta(P-\mathrm{ET})_{\mathrm{obs}}$ is greater than what is expected from natural climate variability; thus, the null hypothesis is rejected. When evaluating spatial pattern agreement in the sign of $\Delta(P-\mathrm{ET})$ as opposed to the correlation, the corresponding probabilities are greater than $98 \%$ for both the DDM and LSM reconstructions. In addition, the agreement between observations and the multimodel mean disappears when replicating the analysis for historical simulations that include natural radiative forcing but no human-induced forcing (Fig. 2b), suggesting that the considered climate models capture the observed change only if human influence is taken into account. Furthermore, these results are generally confirmed when analysing each LSM reconstruction individually, as opposed to the mean reconstruction (Extended Data Table 2). In addition, there is one reconstruction available that uses cruNcep ${ }^{32}$ as atmospheric forcing data (that is, CNRM-CM6-1-cruNcep) instead of the GSWP3 forcing. In this case, the level of agreement with the historical estimate from coupled climate models is also greater than what can be expected from natural climate variability with a probability of $92 \%$ ( $99.7 \%$ when analysing pattern agreement in the sign of the changes).

Individual changes in precipitation and evapotranspiration The observed changes in dry-season water availability $\Delta(P-\mathrm{ET})$ result from changes in precipitation $\Delta P$ and evapotranspiration $\Delta \mathrm{ET}$, since $\Delta(P-\mathrm{ET})=\Delta P-\Delta \mathrm{ET}$. Figure 3 shows the separate contributions of these two terms for the DDM and LSM reconstructions, as well as for the multimodel mean of climate model simulations with historical human-induced plus natural radiative forcing. According to both the DDM and LSM reconstructions, $\Delta$ ET favoured a decrease in available water on $64 \%$ of the land area, whereas this is the case on only $41 \%$ of the land for $\Delta P$. Corresponding values from historical climate simulations are very similar, with $63 \%$ of land where $\Delta \mathrm{ET}$ favours a decrease in water availability and $44 \%$ of land where $\Delta P$ does. At extratropical latitudes, in particular, it is clear that the decrease in dry-season $P$-ET is driven mainly by ET rates that outpace the increase in $P$ (Fig. 4). At these latitudes, on the basis of the DDM (LSM) reconstruction, ET increases on $79 \%(86 \%)$ of the area with negative $\Delta(P-\mathrm{ET})$ and contributes more to the drying than $P$ on $72 \%$ (74\%) of it, highlighting the dominant role ET played to decrease water availability. Corresponding percentages from historical climate simulations are even higher. However, within the tropics, the northern Andes and central Africa are noteworthy regions where decreases in $P$ contribute to less available water during the dry season. In addition, we note that it is highly unlikely that ET would increase in locations where $P$ decreases, since ET can be water limited; $\Delta P$ and $\Delta$ ET show the same sign in $86 \%$ of all grid cells for the DDM reconstruction, in $72 \%$ for the LSM reconstruction and in $78 \%$ for the climate models mean. Overall, our results portray how increased ET contributed to drier dry seasons, which is supported by other studies that highlight the importance of ET for the onset and amplification of drought ${ }^{33,34}$. This is also consistent with increasing atmospheric water demand being more evenly distributed in space and time compared with changes in $P$. Meanwhile, it has been suggested that soil moisture limitation has caused a decline in the annual land ET trend ${ }^{35}$.

In conclusion, there are multiple lines of evidence indicating that changes in dry-season water availability since the first half of the twentieth century are attributable to human-induced climate change. Our results are based on new reconstructions of terrestrial water dynamics from data-driven and land-surface models, together with historical climate model simulations. The fact that climate models only reproduce a pattern similar to the reconstructed changes in water availability when accounting for human-induced forcing indicates that it is highly unlikely that potential uncertainties in the reconstructions could meaningfully alter our conclusions. Moreover, the reconstructions and historical climate model simulations do not agree only on water availability changes, but also on the underlying contributions of $P$ and ET to the changes (Fig. 4). The regional changes in available water during the driest month of the warm growing season are likely to have also affected the energy and carbon cycles through their link with the water cycle. Consistent with drying in extratropical latitudes, in recent years we have experienced more-frequent and widespread hot extremes ${ }^{36}$ possibly amplified by the coupling between soil moisture and temperature ${ }^{37}$. 

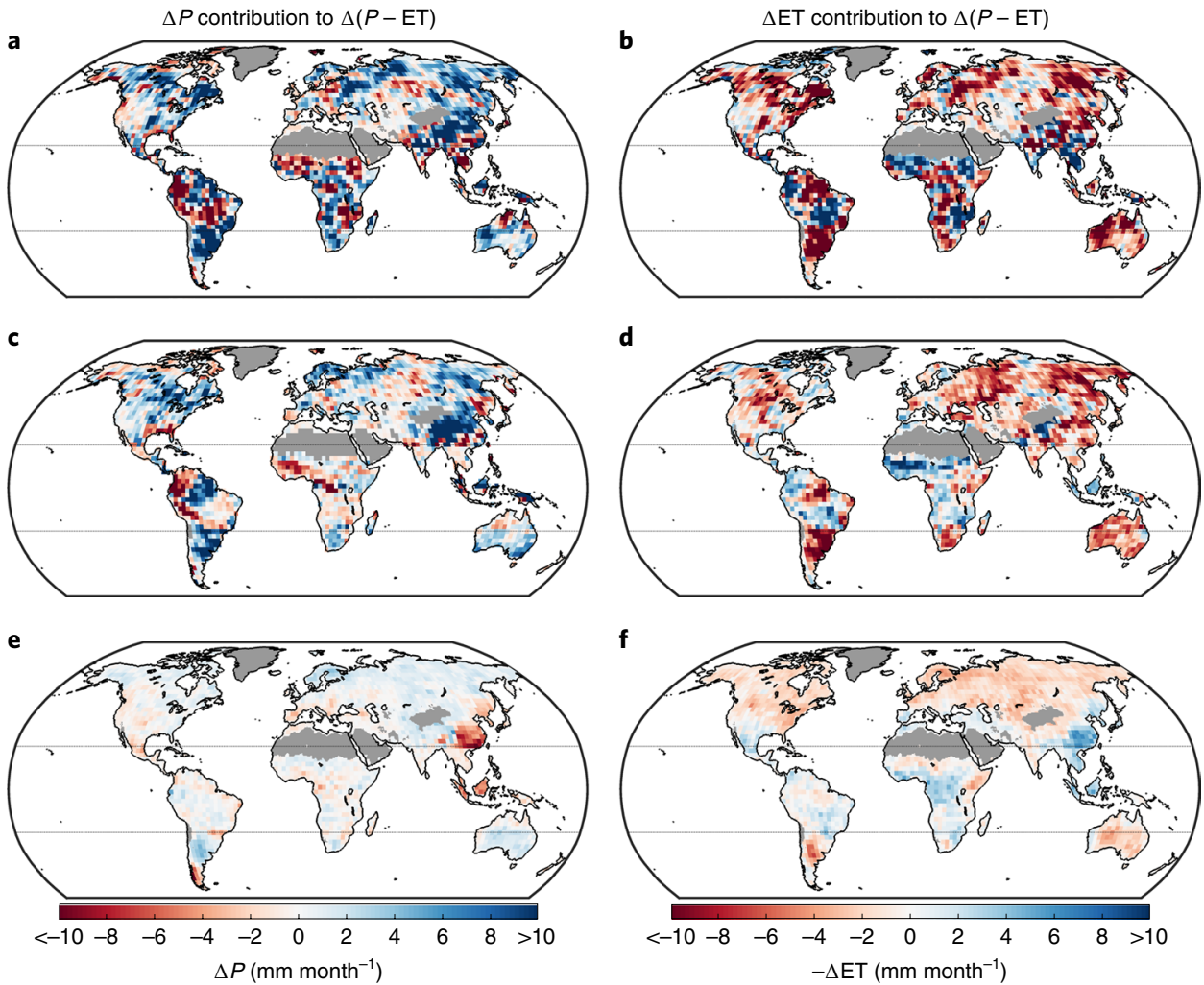

Fig. 3 | Contribution of changes in precipitation and evapotranspiration to changes in dry-season water availability. a,b, DDM reconstruction. c,d, LSM reconstruction. e,f, Multimodel mean of climate model simulations with historical human-induced plus natural radiative forcing. a,c,e, Changes in precipitation $(\Delta P)$. b, d,f, Negative changes in evapotranspiration $(-\Delta \mathrm{ET})$. Blue indicates a contribution to increase water availability and red to decrease it. Antarctica, Greenland and desert regions with annual $P$ below $100 \mathrm{~mm}$ are masked in grey.
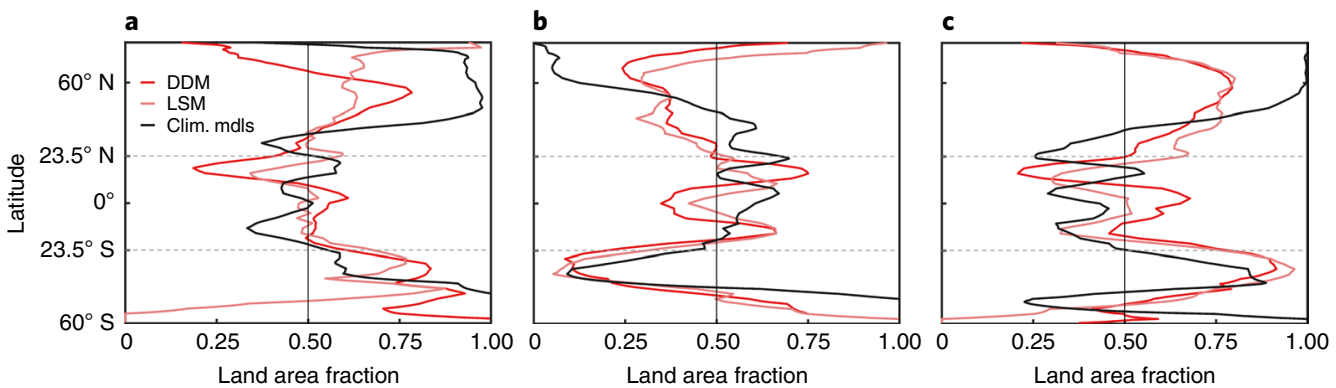

Fig. 4 | Summary of average latitudinal changes in dry-season water availability, precipitation and evapotranspiration. a, Land area fraction with decreasing water availability $(\Delta(P-E T)<0)$. b. Land area fraction with decreasing precipitation $(\Delta P<0)$. $\mathbf{c}$, Land area fraction with increasing evapotranspiration $(\Delta E T>0)$. All panels show changes according to the DDM and LSM observation-based reconstructions, as well as for the multimodel mean of climate model simulations with historical human-induced plus natural radiative forcing. Antarctica, Greenland and desert regions with annual $P$ below $100 \mathrm{~mm}$ are omitted when computing the averages.

In addition, since vegetation productivity is sensitive to water availability $^{38}$, observed dry-season changes could have influenced the land carbon $\sin ^{39}$. The observed regional tendencies are projected to continue under further global warming, highlighting the need for timely climate action.

\section{Online content}

Any methods, additional references, Nature Research reporting summaries, source data, extended data, supplementary information, acknowledgements, peer review information; details of author contributions and competing interests; and statements of data and code availability are available at https://doi.org/10.1038/ s41561-020-0594-1.

Received: 19 August 2019; Accepted: 18 May 2020;

Published online: 29 June 2020

\section{References}

1. Seneviratne, S. I. et al. in Managing the Risks of Extreme Events and Disasters to Advance Climate Change Adaptation (eds Field, C. B. et al.) 109-230 (Cambridge Univ. Press, 2012).

2. Collins, M. et al. in Climate Change 2013: The Physical Science Basis (eds Stocker, T. F. et al.) 1029-1136 (IPCC, Cambridge Univ. Press, 2013). 
3. Hoegh-Guldberg, O. et al. in Special Report on Global Warming of $1.5^{\circ} \mathrm{C}$ (eds Masson-Delmotte, V. et al.) Ch. 3 (WMO, 2018).

4. Pendergrass, A. G. \& Knutti, R. The uneven nature of daily precipitation and its change. Geophys. Res. Lett. 45, 980-11,988 (2018).

5. Greve, P., Roderick, M. L. \& Seneviratne, S. I. Simulated changes in aridity from the last glacial maximum to $4 \mathrm{xCO}_{2}$. Environ. Res. Lett. 12, 114021 (2017).

6. Greve, P., Gudmundsson, L. \& Seneviratne, S. I. Regional scaling of annual mean precipitation and water availability with global temperature change. Earth Syst. Dyn. 9, 227-240 (2018).

7. Kumar, S., Lawrence, D. M., Dirmeyer, P. A. \& Sheffield, J. Less reliable water availability in the 21st century climate projections. Earth Future 2, 152-160 (2013).

8. Kumar, S., Allan, R. P., Zwiers, F., Lawrence, D. M. \& Dirmeyer, P. A. Revisiting trends in wetness and dryness in the presence of internal climate variability and water limitations over land. Geophys. Res. Lett. 42, 10867-10875 (2015)

9. Orlowsky, B. \& Seneviratne, S. I. Elusive drought: uncertainty in observed trends and short- and long-term CMIP5 projections. Hydrol. Earth Syst. Sci. 17, 1765-1781 (2013).

10. Marvel, K. et al. Twentieth-century hydroclimate changes consistent with human influence. Nature 569, 59-65 (2019).

11. Zhang, X. et al. Detection of human influence on twentieth-century precipitation trends. Nature 448, 461-465 (2007).

12. Marvel, K. \& Bonfils, C. Identifying external influences on global precipitation. Proc. Natl Acad. Sci. USA 110, 301-19,306 (2013).

13. Douville, H., Ribes, A., Decharme, B., Alkama, R. \& Sheffield, J. Anthropogenic influence on multidecadal changes in reconstructed global evapotranspiration. Nat. Clim. Change 3, 59-62 (2013).

14. Gudmundsson, L., Seneviratne, S. I. \& Zhang, X. Anthropogenic climate change detected in European renewable freshwater resources. Nat. Clim. Change 7, 813-816 (2017).

15. Gu, X. et al. Attribution of global soil moisture drying to human activities: quantitative viewpoint. Geophys. Res. Lett. 46, 2573-2582 (2019).

16. Palmer, W. C. Meteorological Drought Research Paper No. 45 (Department of Commerce, 1965).

17. Dai, A. Drought under global warming: a review. WIREs Clim. Change 2, 45-65 (2010).

18. Briffa, K. R., van der Schrier, G. \& Jones, P. D. Wet and dry summers in Europe since 1750: evidence of increasing drought. Int. J. Climatol. 29 1894-1905 (2009).

19. Sheffield, J., Wood, E. F. \& Roderick, M. L. Little change in global drought over the past 60 years. Nature 491, 435-438 (2012).

20. Roderick, M. L., Greve, P. \& Farquhar, G. D. On the assessment of aridity with changes in atmospheric $\mathrm{CO}_{2}$. Water Resour. Res. 51, 5450-5463 (2015).

21. Milly, P. C. D. \& Dunne, K. A. Potential evapotranspiration and continental drying. Nat. Clim. Change 6, 946-949 (2016).

22. Held, I. \& Soden, B. Robust responses of the hydrological cycle to global warming. J. Clim. 19, 5686-5699 (2006).
23. Greve, P. et al. Global assessment of trends in wetting and drying over land. Nat. Geosci. 7, 716-721 (2014).

24. Byrne, M. P. \& O'gorman, P. A. The response of precipitation minus evapotranspiration to climate warming: why the "wet-get-wetter, dry-get-drier"' scaling does not hold over land. J. Clim. 28, 8078-8092 (2015).

25 . Chou, C. et al. Increase in the range between wet and dry season precipitation. Nat. Geosci. 6, 263-267 (2013).

26. Boisier, J. P., Ciais, P., Ducharne, A. \& Guimberteau, M. Projected strengthening of Amazonian dry season by constrained climate model simulations. Nat. Clim. Change 5, 656-660 (2015).

27. Ghiggi, G., Humphrey, V., Seneviratne, S. I. \& Gudmundsson, L. GRUN: an observation-based global gridded runoff dataset from 1902 to 2014. Earth Syst. Sci. Data 11, 1655-1674 (2019).

28. Humphrey, V. \& Gudmundsson, L. GRACE-REC: a reconstruction of climate-driven water storage changes over the last century. Earth Syst. Sci. Data 11, 1153-1170 (2019).

29. van den Hurk, B. et al. LS3MIP (v1.0) contribution to CMIP6: the Land Surface, Snow and Soil moisture Model Intercomparison Project-aims, setup and expected outcome. Geosci. Model Dev. 9, 2809-2832 (2016).

30. Kim, H. J. Global Soil Wetness Project Phase 3 Atmospheric Boundary Conditions (Experiment 1) (DIAS, 2017).

31. Wilcox, L. J., Highwood, E. J. \& Dunstone, N. J. The influence of anthropogenic aerosol on multi-decadal variations of historical global climate. Environ. Res. Lett. 8, 024033 (2013).

32. Viovy, N. CRUNCEP Version 7-Atmospheric Forcing Data for the Community Land Model (NCAR, 2018).

33. Seneviratne, S. I. et al. Swiss prealpine Rietholzbach research catchment and lysimeter: 32 year time series and 2003 drought event. Water Resour. Res. 48, W06526 (2012)

34. Teuling, A. J. et al. Evapotranspiration amplifies European summer drought. Geophys. Res. Lett. 40, 2071-2075 (2013).

35. Jung, M. et al. Recent decline in the global land evapotranspiration trend due to limited moisture supply. Nature 467, 951-954 (2010).

36. Vogel, M. M., Zscheischler, J., Wartenburger, R., Dee, D. \& Seneviratne, S. I. Concurrent 2018 hot extremes across Northern Hemisphere due to human-induced climate change. Earth Future 7, 692-703 (2019).

37. Seneviratne, S. I. et al. Investigating soil moisture-climate interactions in a changing climate: a review. Earth Sci. Rev. 99, 125-161 (2010).

38. Humphrey, V. et al. Sensitivity of atmospheric $\mathrm{CO}_{2}$ growth rate to observed changes in terrestrial water storage. Nature 560, 628-631 (2018).

39. Murray-Tortarolo, G. et al. The dry season intensity as a key driver of NPP trends. Geophys. Res. Lett. 43, 2632-2639 (2016).

Publisher's note Springer Nature remains neutral with regard to jurisdictional claims in published maps and institutional affiliations.

(C) The Author(s), under exclusive licence to Springer Nature Limited 2020 


\section{Methods}

Changes in dry-season water availability. The definition of water available to society corresponds to that of renewable freshwater resources given by the long-term average flow of streams and groundwater, that is, $R+\Delta \mathrm{TWS}$, which is equivalent to $P$-ET on the basis of the water balance. Therefore, here we define dry-season water availability as annual minimum monthly $P$-ET, similar to previous studies ${ }^{7,8}$. Note that this definition does not include the actual magnitude of TWS, which is affected by $P$ and ET from preceding months.

For the DDM reconstruction of water availability we use $R$ and $\Delta$ TWS estimates $(P-\mathrm{ET}=R+\Delta$ TWS $)$ from two new reconstructions calibrated with observations, which perform well compared with state-of-the-art hydrological model ${ }^{27,28}$. The reconstructions employ $P$ and near-surface air temperature data as explanatory variables. It could be that potential additional influences on past continental water availability, for example land use changes and the response of vegetation to increasing atmospheric $\mathrm{CO}_{2}^{40,41}$, are not fully captured in these reconstructions. In addition, we employ $P$-ET data from six LSMs that are driven with observational atmospheric GSWP $3^{30}$ data and correspond to the Land Surface, Snow and Soil Moisture Model Intercomparison Project (LS3MIP) ${ }^{29}$. The LSMs account for land use changes but do not include local land-atmosphere feedbacks. Note that Extended Data Table 2 also includes results from a single LSM reconstruction driven with observational atmospheric data from cruNcep ${ }^{32}$. These simulations are interpolated to a common $2.5^{\circ} \times 2.5^{\circ}$ grid using second-order conservative remapping.

Monthly $P$-ET data are available during the period 1902-2014. For each year in this period, we identify the month with the lowest $P$-ET; that is, one value of $(P-\mathrm{ET})_{\min }$ per year $y$. For grid cells within tropical latitudes $\left(\leq 23.5^{\circ}\right)$, we identify the month with minimum $P$-ET from all 12 months of each year, whereas in extratropical latitudes $\left(>23.5^{\circ}\right)$, we identify the month with minimum $P$-ET only from the period May-September in the Northern Hemisphere and from the period November-March in the Southern Hemisphere. We consider these periods to represent warmer months within the active vegetation season. Next we compute at each grid cell the average of $(P-E T)_{\min }$ during the recent period 1985-2014 as:

$$
(P-\mathrm{ET})_{\text {pres }}=\frac{1}{30} \sum_{y=1985}^{y=2014}(P-\mathrm{ET})_{\min , y}
$$

and correspondingly during the reference period $1902-1950$ as:

$$
(P-\mathrm{ET})_{\text {past }}=\frac{1}{49} \sum_{y=1902}^{y=1950}(P-\mathrm{ET})_{\min , y}
$$

Last, we define the change in dry-season water availability as the difference between the recent and reference periods:

$$
\Delta(P-\mathrm{ET})=(P-\mathrm{ET})_{\text {pres }}-(P-\mathrm{ET})_{\text {past }}
$$

We consider a multidecadal present and past period to reduce the influence of natural short-term interannual variability on the analysed change. Extended Data Fig. 3 shows the sensitivity of the observed change to the definition of the reference period.

Land area fraction. Data are available in a regular longitude-latitude grid. Therefore, grid cells do not have an equal size, with smaller grid cells at higher latitudes. Hence, for calculating the land area fractions, we must assign a weight to each grid cell on the basis of size. Here we compute these weights $\left(w_{i}\right)$ as the size of each grid cell at latitude lat $_{i}$ relative to the size of the largest grid cells located at the Equator, given by:

$$
W_{i}=\left|\frac{\sin \left(\text { lat }_{i}+(0.5 \times \text { res })\right)-\sin \left(\text { lat }_{i}-(0.5 \times \text { res })\right)}{\sin (0.5 \times \text { res })-\sin (-0.5 \times \text { res })}\right|
$$

where lat is the vector indicating the latitude of each grid cell centre, and ranges from $-90+(0.5 \times$ res $)$ to $90-(0.5 \times$ res $)$ with increasing step or resolution denoted by res.

Attribution of changes in dry-season water availability to human-induced climate change. Here we follow a simple attribution approach based on a correlation analysis using observation-based reconstructions (obs) and different climate model simulations $(\mathrm{mdl})^{14,42}$. Employed simulations from the fifth phase of the Coupled Model Intercomparison Project (CMIP5) are bilinearly interpolated to a common $2.5^{\circ} \times 2.5^{\circ}$ grid and detailed in Extended Data Table 1. They include historical simulations (hist) with radiative forcing from both human-induced emissions (greenhouse gases and aerosols) and natural forcing (volcanic and solar activity), historical simulations (histNat) with natural forcing only, and simulations with greenhouse gas levels set to pre-industrial conditions $(\mathrm{pi})^{43}$. Both hist and histNat simulations end in 2005 . We extend hist simulations with those from the Representative Concentration Pathway 8.5 (RCP 8.5) ${ }^{44}$ until 2014 to match the observational record period. Following the same procedure as for the reconstructions, we obtain for each model the change in dry-season water availability as the difference between equation (1) and equation (2). The multimodel mean is denoted as $\Delta(P-\mathrm{ET})_{\text {mdl,hist }}$. We also obtain $\Delta(P-\mathrm{ET})_{\text {mdl,histNat }}$.
However, note that because there are no data to extend histNat simulations, equation (1) is adjusted as follows:

$$
(P-\mathrm{ET})_{\mathrm{pres}}=\frac{1}{21} \sum_{y=1985}^{y=2005}(P-\mathrm{ET})_{\min , y}
$$

In addition, we obtain 320 estimates of changes in dry-season water availability from pi simulations $\left(\Delta(P-\mathrm{ET})_{\text {mdl,pi }}\right)$ by considering 113 -yr periods and computing the difference between the last 30 years and the first 49 years of the period, as for the observations. Given that these pi simulations do not include additional forcing from greenhouse gas emissions, they represent possible changes resulting only from natural climate variability. Because of model resolution, the latitudinal tropical boundary is set at $22.5^{\circ}$ instead of $23.5^{\circ}$. Observed changes denoted by $\Delta(P-\mathrm{ET})_{\mathrm{obs}}$ are recomputed after averaging to a coarser $2.5^{\circ}$ grid resolution to match the model data. Note that when comparing $\Delta(P-\mathrm{ET})_{\text {mdl,histNat }}$ with $\Delta(P-\mathrm{ET})_{\mathrm{obs}}$, the changes are between the recent period 1985-2005 (instead of 1985-2014) and the reference period 1902-1950.

The logic behind our attribution methodology is as follows:

1. The multimodel mean of coupled simulations with historical forcing (mdl,hist) estimates the response of the climate system to the external forcing given by human-induced climate change.

2. The spatial correlation between this multimodel mean and the observations, that is corr(mdl,hist and obs), quantifies the similarity between the observed response and the estimated response to human-induced climate change.

3. The null hypothesis is that there is no signal in the observations resulting from human-induced climate change, and therefore corr(mdl,hist and obs) is only a consequence of natural climate variability ( $\mathrm{ncv}$ ).

4. To test this hypothesis, we need estimates of possible responses of the climate system arising only from ncv to obtain a distribution of corr(mdl,hist and ncv).

5. The ncv estimates are approximated using coupled climate model simulations without any of the external forcing from human-induced climate change, also referred to as simulations forced with pre-industrial conditions (pi).

6. If corr(mdl,hist and obs) is greater than almost all the estimates of corr(mdl,hist and ncv), then the null hypothesis is rejected with high confidence. This indicates that the observed response includes a signal stemming from the external forcing given by human-induced climate change.

7. Furthermore, if the null hypothesis cannot be rejected when using the multimodel mean of coupled simulations with only historical natural forcing (mdl,histNat) as opposed to full historical forcing (mdl,hist), then the influence of human-induced climate change on the observed response is confirmed.

In summary, if the spatial Spearman correlation between $\Delta(P-\mathrm{ET})_{\mathrm{mdl}, \text { hist }}$ and $\Delta(P-\mathrm{ET})_{\mathrm{obs}}$ is greater than what is expected from natural climate variability (that is, correlations between $\Delta(P-\mathrm{ET})_{\text {mdl,hist }}$ and each of the hundreds of estimates of $\left.\Delta(P-\mathrm{ET})_{\mathrm{mdlp}}\right)$, then we could conclude that a response to the historical forcing is detectable in the observed pattern. Next, if the spatial Spearman correlation between $\Delta(P-\mathrm{ET})_{\text {mdl,histNat }}$ and $\Delta(P-\mathrm{ET})_{\mathrm{obs}}$ is not different than what is expected from natural climate variability (that is, correlations between $\Delta(P-\mathrm{ET})_{\text {mdl,histNat }}$ and estimates of $\left.\Delta(P-\mathrm{ET})_{\mathrm{mdl}, \mathrm{pi}}\right)$, then it is unlikely that historical natural radiative forcing is causing the observed pattern. Thus, it would follow that it is highly likely that human-induced climate change is causing the pattern of observed changes in dry-season water availability.

Contribution of changes in $\boldsymbol{P}$ and ET to changes in dry-season water availability. Changes in dry-season water availability given by equation (3) result from changes in both $P$ and ET, as follows:

$$
\Delta(P-\mathrm{ET})=\Delta P-\Delta \mathrm{ET}
$$

Increases in $P$ and decreases in ET favour an increase in dry-season water availability, whereas decreases in $P$ and increases in ET favour a decrease in available water. For the DDM reconstruction, we use $P$ data from GSWP $3^{30}$ and derive ET from the water balance ET $=P-R-\Delta$ TWS. Conversely, the LSM reconstructions directly estimate ET and, therefore, avoid the potential for error amplification, which arises when computing ET as a residual. As described in the preceding, there is one value of $(P-\mathrm{ET})_{\min }$ per year corresponding to the month with the lowest $P$-ET. $P_{\min }$ and $\mathrm{ET}_{\min }$ then correspond to the values from this month. By replacing $(P-\mathrm{ET})$ in equations (1) and (2) with $\mathrm{P}$ and ET separately, we obtain $P_{\text {past }}, P_{\text {pres }}, \mathrm{ET}_{\text {past }}$ and $\mathrm{ET}_{\text {pres. }}$. The contributions of changes in $P$ and ET to changes in dry-season water availability are computed as:

$$
\begin{gathered}
\Delta P=P_{\text {pres }}-P_{\text {past }} \\
\Delta \mathrm{ET}=\mathrm{ET}_{\text {pres }}-\mathrm{ET}_{\text {past }}
\end{gathered}
$$

\section{Data availability}

Precipitation data from the Global Soil Wetness Project Phase 3 (GSWP-3) are available at https://doi.org/10.20783/DIAS.501. The runoff reconstruction 
dataset GRUN is available at https://doi.org/10.3929/ethz-b-000324386, and the reconstruction of changes in terrestrial water storage is available at https://doi. org $/ 10.6084 / \mathrm{m} 9$.figshare.7670849. The land-surface model reconstructions and CMIP5 climate model data used in this study are available at https://esgf-node.llnl. gov/projects/esgf-llnl/.

\section{References}

40. Piao, S. et al. Changes in climate and land use have a larger direct impact than rising $\mathrm{CO}_{2}$ on global river runoff trends. Proc. Natl Acad. Sci. USA 104, 242-15,247 (2007)

41. Ukkola, A. M. et al. Reduced streamflow in water-stressed climates consistent with $\mathrm{CO}_{2}$ effects on vegetation. Nat. Clim. Change 6, 75-78 (2016).

42. Bindoff, N. L. et al. in Climate Change 2013: The Physical Science Basis (eds Stocker, T. F. et al.) Ch. 10 (IPCC, Cambridge Univ. Press, 2013).

43. Taylor, K. E. et al. An overview of CMIP5 and the experiment design. Bull. Am. Meteorol. Soc. 93, 485-498 (2012).

44. Moss, R. H. et al. The next generation of scenarios for climate change research and assessment. Nature 463, 747-756 (2010).

\section{Acknowledgements}

R.S.P., L.G. and S.I.S. acknowledge partial support from the European Research Council (ERC) DROUGHT-HEAT project funded by the European Community's Seventh Framework Programme (grant agreement FP7-IDEAS-ERC-617518) and from the European Union's Horizon 2020 Research and Innovation Program (grant agreement 821003 (4C)). D.M.L. was supported in part by the Reducing Uncertainties in Biogeochemical Interactions through Synthesis and Computing Scientific Focus Area (RUBISCO SFA), which is sponsored by the Regional and Global Climate Modeling (RGCM) Program in the US Department of Energy Office of Science. J.M. was also supported by the RUBISCO SFA. Oak Ridge National Laboratory is managed by UT-BATTELLE for DOE under contract number DE-AC05-00OR22725. D.P. acknowledges the European Union's Horizon 2020 research and innovation program under Grant
Agreement 641816 (CRESCENDO) that partially funded the CMCC simulations. H.K. acknowledges Grant-in-Aid for Specially Promoted Research 16H06291 and 18KK0117 from Japan Society for the Promotion of Science. The LS3MIP simulation of the Institut Pierre Simon Laplace (IPSL) was performed at the Très Grand Centre de Calcul (TGCC) under the allocation 2018- R0040110492 (project gencmip6) provided by GENCI (Grand Equipement National de Calcul Intensif). We acknowledge the World Climate Research Program's Working Group on Coupled Modelling, which is responsible for the Coupled Model Intercomparison Project (CMIP), and we thank the climate modelling groups for producing and making available their model output. For CMIP, the US Department of Energy's Program for Climate Model Diagnosis and Intercomparison provides coordinating support and led development of software infrastructure in partnership with the Global Organization for Earth System Science Portals. We thank U. Beyerle, J. Sedlacek and L. Brunner for downloading and processing the CMIP5 and LS3MIP data.

\section{Author contributions}

R.S.P., L.G. and S.I.S. designed the study. R.S.P. performed the analysis and wrote the manuscript. B.D., A.D., D.M.L., J.M. and D.P. contributed to the land-surface model reconstructions. G.K., S.I.S. and H.K. coordinated the land-surface model experiments. H.K. produced the forcing dataset for the data-driven and land-surface model reconstructions. All authors discussed the results and read and reviewed the manuscript.

\section{Competing interests}

The authors declare no competing interests.

\section{Additional information}

Extended data is available for this paper at https://doi.org/10.1038/s41561-020-0594-1.

Correspondence and requests for materials should be addressed to R.S.P.

Peer review information Primary Handling Editors: Tamara Goldin; Heike Langenberg

Reprints and permissions information is available at www.nature.com/reprints. 


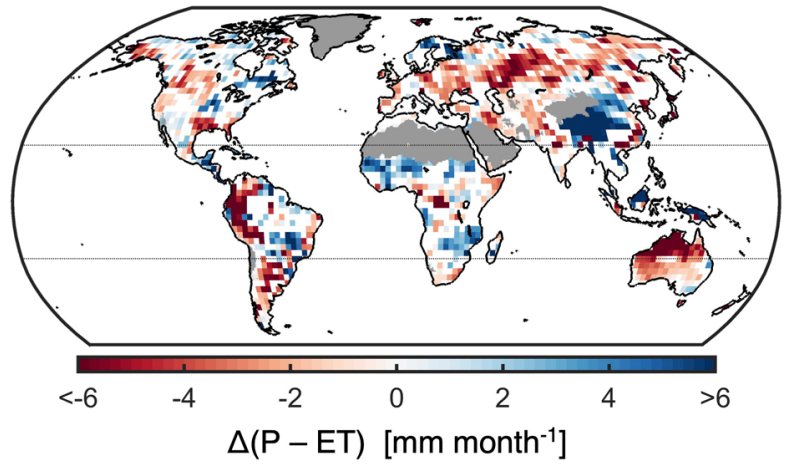

Extended Data Fig. 1 | Agreement between reconstructions from data driven (DDM) and land surface models (LSM). Mean change in dry season water availability from the DDM and LSM reconstructions (that is mean of Fig. 1a, b of the main article). Shown are only grid cells where both reconstructions agree on the sign of change. Grey lines indicate tropical boundaries at $23.5^{\circ} \mathrm{S}$ and $23.5^{\circ} \mathrm{N}$. Antarctica, Greenland and desert regions with annual P below $100 \mathrm{~mm}$ are masked in grey. 


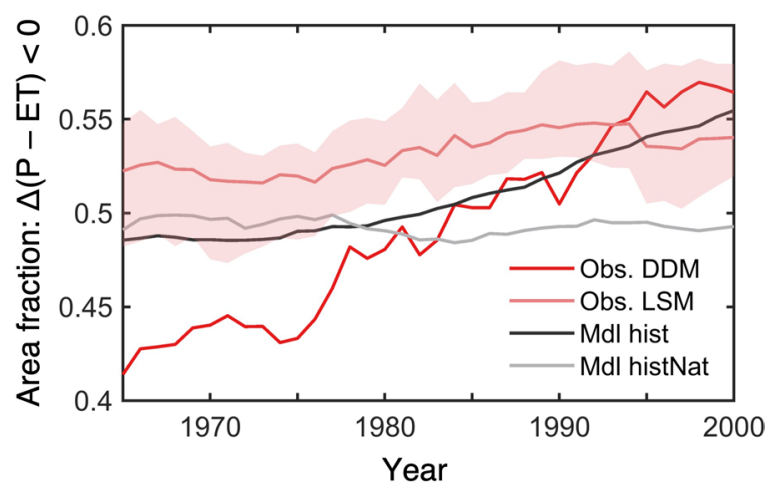

Extended Data Fig. 2 | Temporal evolution of land area fraction with decrease in dry season water availability. $\Delta(P-E T)$ is obtained as the difference between average P - ET from a 30-year period centered around the indicated year and average P - ET from the reference period 1902-1950. Lines indicate the DDM estimate, as well as the mean of individual LSM reconstructions and the mean of individual climate model simulations. The shaded area indicates the ensemble range of the 6 individual LSM reconstructions. The ensemble range of individual climate model simulations is not shown, but in the most recent period corresponds to $0.42-0.71$ for models with full historical forcing (hist) and $0.46-0.54$ for models with only natural historical forcing (histNat). Antarctica, Greenland and desert regions with annual $\mathrm{P}$ below $100 \mathrm{~mm}$ are omitted. 
Reconstruction from data-driven models (DDM)

Reconstruction from land surface models (LSM)

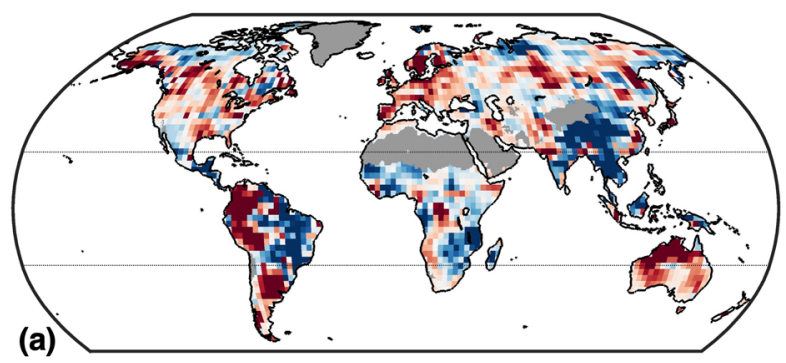

(a)
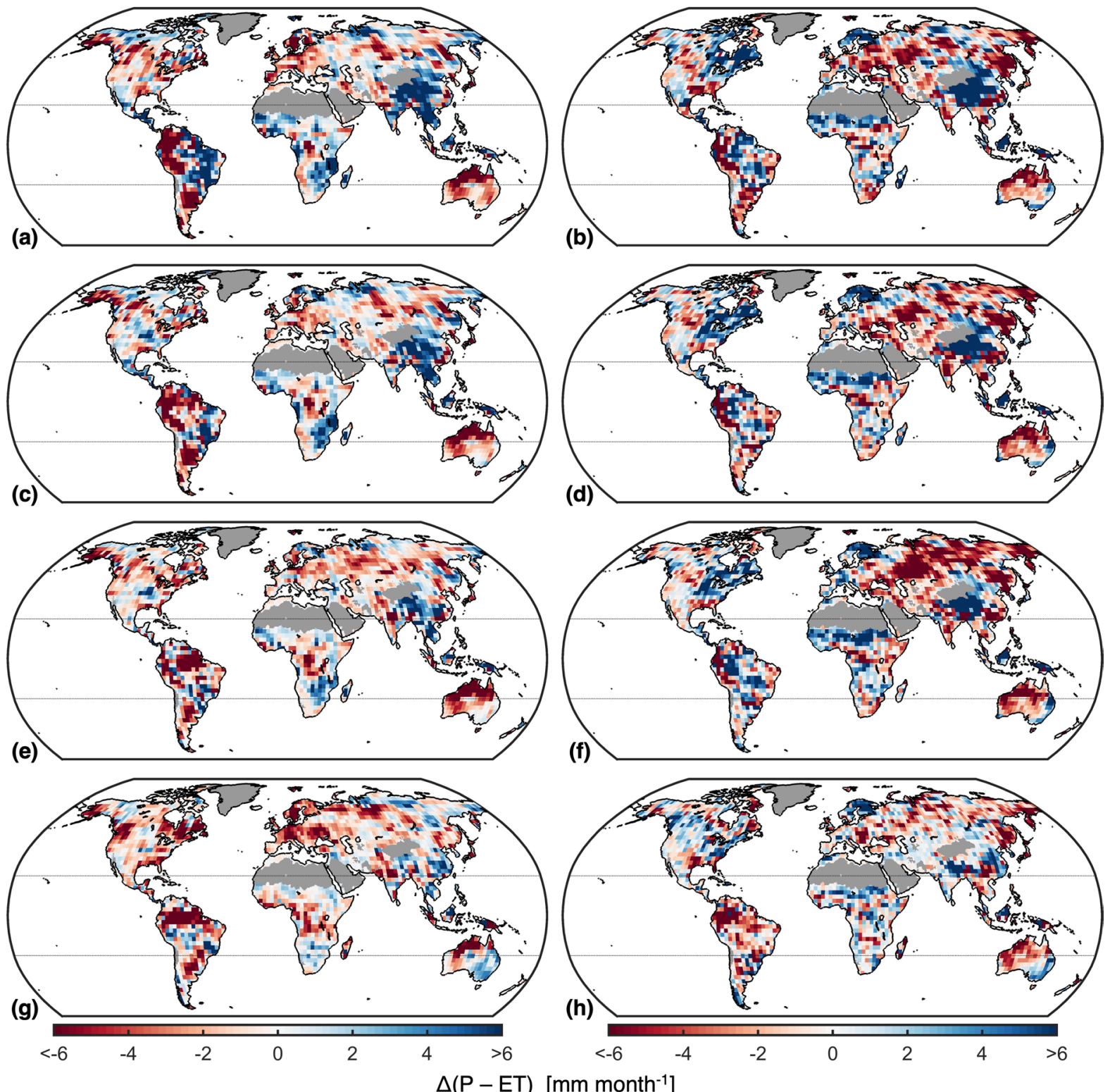

Extended Data Fig. 3 | Sensitivity to the definition of the reference time period. The reference period considered in Fig. 1 of the article is 1902-1950, whereas here we consider 4 alternative options: (a, b) 1902-1930, (c, d) 1911-1940, (e, f) 1921-1950 and (g, h) 1951-1980. Note that during the period 1951-1980 the influence of aerosol emissions was relatively high. Plots from the DDM reconstruction are shown on the left (a, $\mathbf{c}, \mathbf{e}, \mathbf{g})$ and plots from the $\mathrm{LSM}$ reconstruction on the right $(\mathbf{b}, \mathbf{d}, \mathbf{f}, \mathbf{h})$. Grey lines indicate tropical boundaries at $23.5^{\circ} \mathrm{S}$ and $23.5^{\circ} \mathrm{N}$. Antarctica, Greenland and desert regions with annual $\mathrm{P}$ below $100 \mathrm{~mm}$ are masked in grey. 

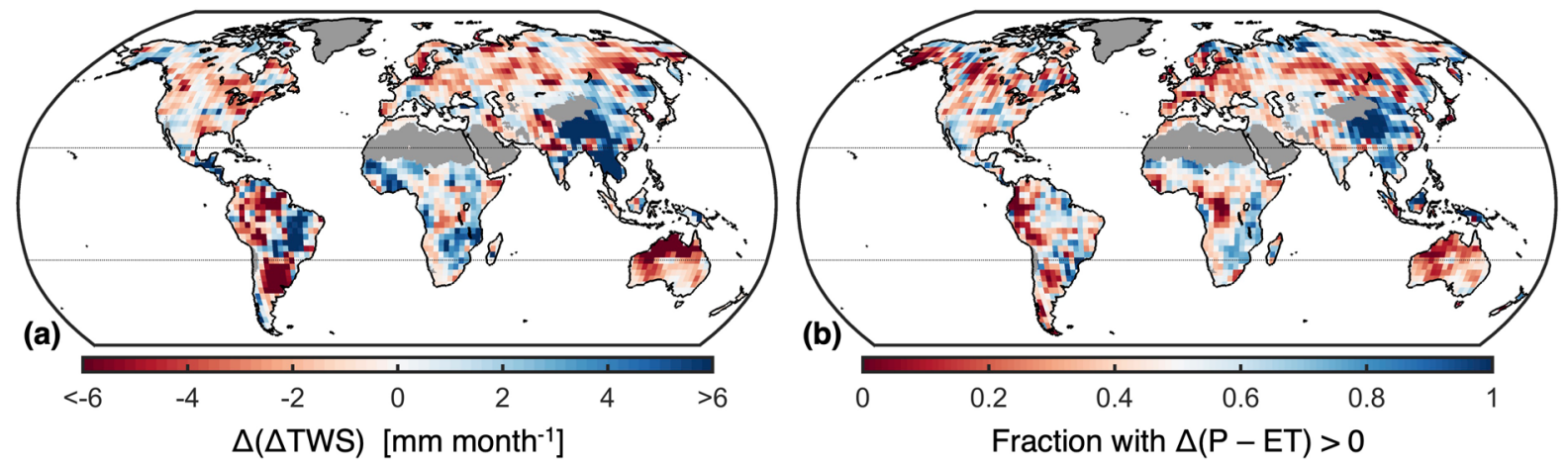

Extended Data Fig. 4 | Contribution of $\Delta$ ( $\Delta$ TWS) to $\Delta$ (P - ET) from the DDM reconstruction (Fig. 1a) and associated uncertainty. a, $\Delta$ ( $\Delta$ TWS) is the difference in average $\Delta$ TWS corresponding to the month with minimum P - ET between years in the periods 1985-2014 and 1902-1950. Note that based on the water balance $\Delta(P-E T)=\Delta(\Delta T W S)+\Delta R$. The $\Delta T$ TW reconstruction used in the article corresponds to the mean of 100 stochastic realizations, whereas no stochastic realizations of the R reconstruction are available. $\mathbf{b}$, Fraction of stochastic realizations of $\Delta(\Delta T$ WS $)$ that result in positive $\Delta(P-E T)$ at each grid cell. Grey lines indicate tropical boundaries at $23.5^{\circ} \mathrm{S}$ and $23.5^{\circ} \mathrm{N}$. Antarctica, Greenland and desert regions with annual $\mathrm{P}$ below $100 \mathrm{~mm}$ are masked in grey. 


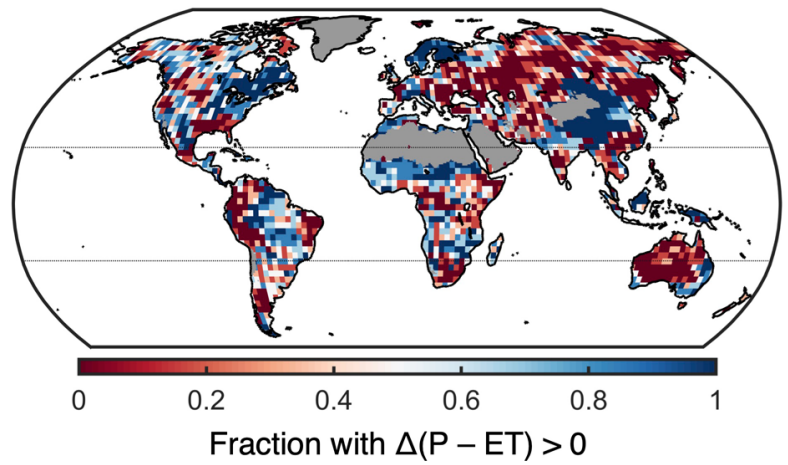

Extended Data Fig. 5 | Agreement between reconstructions from six individual land surface models used for the mean LSM reconstruction (Fig. 1b). Fraction of reconstructions with positive $\Delta(\mathrm{P}-\mathrm{ET})$ at each grid cell. Grey lines indicate tropical boundaries at $23.5^{\circ} \mathrm{S}$ and $23.5^{\circ} \mathrm{N}$. Antarctica, Greenland and desert regions with annual $\mathrm{P}$ below $100 \mathrm{~mm}$ are masked in grey. 
Observation-based data-driven models (DDM)

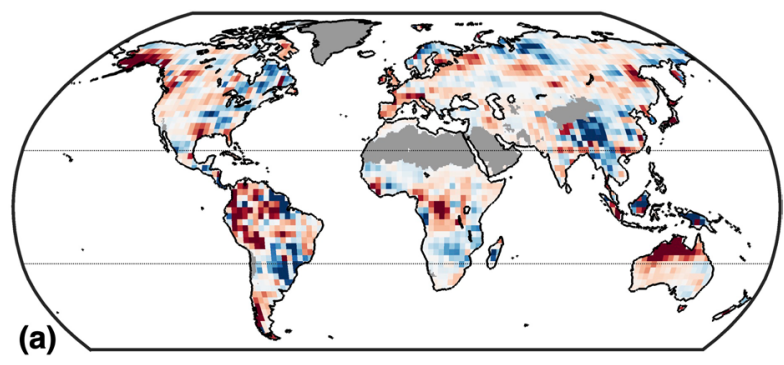

Observation-based land surface models (LSM)

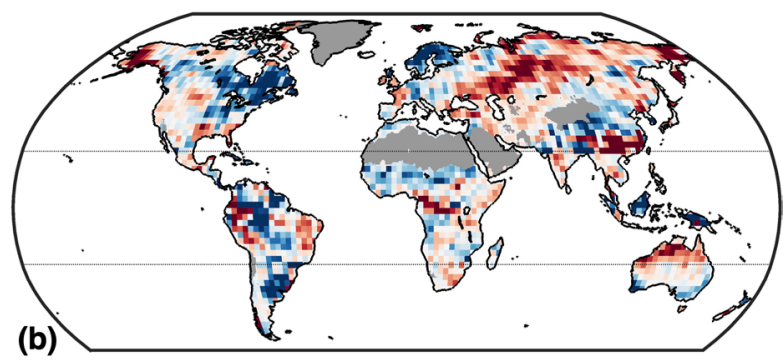

Climate models with full historical forcing

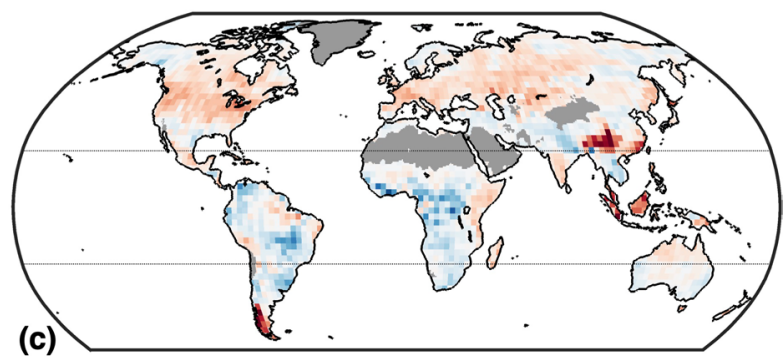

Climate models with only natural historical forcing

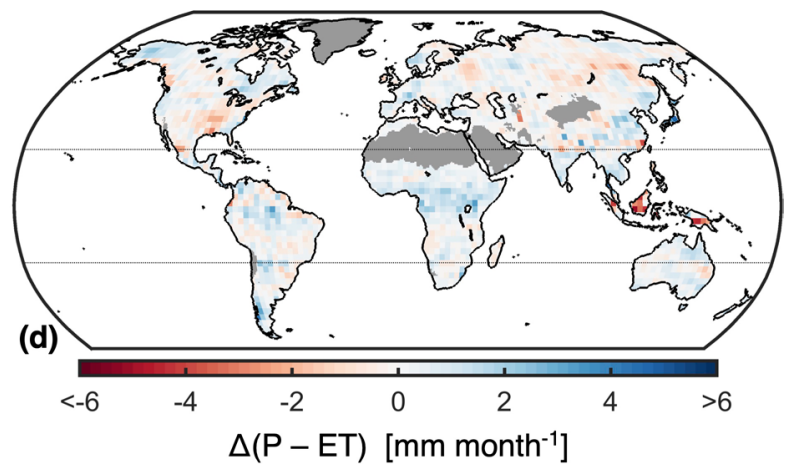

Extended Data Fig. 6 | Sensitivity to the definition of dry season water availability. Dry season water availability is represented by minimum monthly $\mathrm{P}$ - ET in Fig.1 of the article, whereas here we use minimum 3-monthly P - ET. Grey lines indicate tropical boundaries at $23.5^{\circ} \mathrm{S}$ and $23.5^{\circ} \mathrm{N}$. Antarctica, Greenland and desert regions with annual $\mathrm{P}$ below $100 \mathrm{~mm}$ are masked in grey. 
Full historical forcing

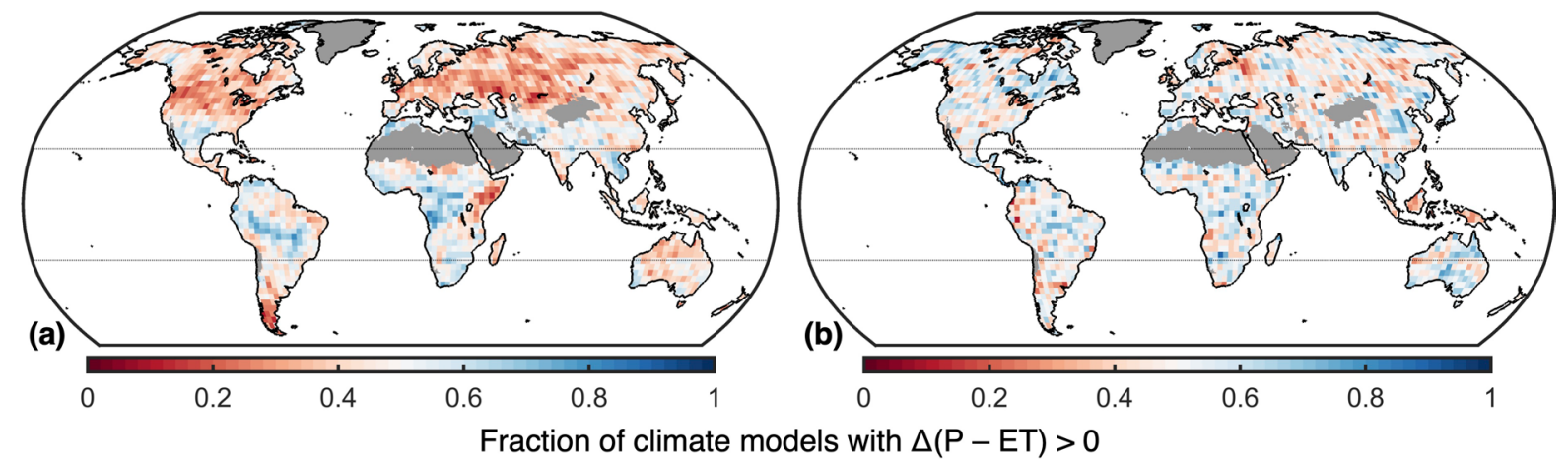

Extended Data Fig. $7 \mid$ Agreement in the sign of $\Delta(\mathrm{P}-\mathrm{ET})$ between individual climate model simulations. Fraction of individual climate model simulations with positive $\Delta(P-E T)$ at each grid cell for (a) simulations with full historical forcing and (b) simulations with only natural historical forcing. Grey lines indicate tropical boundaries at $23.5^{\circ} \mathrm{S}$ and $23.5^{\circ} \mathrm{N}$. Antarctica, Greenland and desert regions with annual P below $100 \mathrm{~mm}$ are masked in grey. 
Extended Data Table 1 | List of climate model simulations

\begin{tabular}{|c|c|c|c|c|c|c|c|}
\hline \multirow{2}{*}{ Model } & \multicolumn{3}{|c|}{ Radiative forcing } & \multirow{2}{*}{ Model } & \multicolumn{3}{|c|}{ Radiative forcing } \\
\hline & hist & histNat & $p i$ & & hist & histNat & $p i$ \\
\hline ACCESS1-0 & $\mathrm{X}$ & & $\mathrm{X}$ & GISS-E2-H-CC & $\mathrm{X}$ & & $\mathrm{X}$ \\
\hline ACCESS1-3 & $X$ & & $X$ & GISS-E2-R & $\mathrm{X}$ & $\mathrm{X}$ & $X^{*}$ \\
\hline BCC-CSM-1 & $X$ & $X$ & $\mathrm{X}$ & GISS-E2-R-CC & $X$ & & $\mathrm{X}$ \\
\hline BCC-CSM1-1-m & $\mathrm{X}$ & & $\mathrm{X}$ & HadGEM2-CC & $\mathrm{X}$ & & $\mathrm{X}$ \\
\hline BNU-ESM & $\mathrm{X}$ & & $\mathrm{X}$ & HadGEM2-ES & $\mathrm{X}$ & $\mathrm{X}$ & \\
\hline CanESM2 & $\mathrm{X}$ & $\mathrm{X}$ & $X$ & INM-CM4 & $\mathrm{X}$ & & $\mathrm{X}$ \\
\hline CCSM4 & $\mathrm{X}$ & $X$ & $\mathrm{X}$ & IPSL-CM5A-LR & $\mathrm{X}$ & $\mathrm{X}$ & $\mathrm{X}$ \\
\hline CESM1-BGC & $X$ & & $\mathrm{X}$ & IPSL-CM5A-MR & $X$ & $\mathrm{X}$ & $\mathrm{X}$ \\
\hline CESM1-CAM5 & $\mathrm{X}$ & $\mathrm{X}$ & $X$ & IPSL-CM5B-LR & $\mathrm{X}$ & & $\mathrm{X}$ \\
\hline CMCC-CESM & & & $\mathrm{X}$ & MIROC-ESM & $\mathrm{X}$ & $X$ & $\mathrm{X}$ \\
\hline CMCC-CM & $X$ & & $X$ & MIROC-ESM-CHEM & $X$ & $X$ & $\mathrm{X}$ \\
\hline CNRM-CM5 & $\mathrm{X}$ & $X$ & $\mathrm{X}$ & MIROC5 & $\mathrm{X}$ & & $\mathrm{X}$ \\
\hline CSIRO-Mk3-6-0 & $\mathrm{X}$ & $\mathrm{X}$ & $\mathrm{X}$ & MPI-ESM-LR & $\mathrm{X}$ & & $\mathrm{X}$ \\
\hline FGOALS-g2 & $\mathrm{X}$ & $\mathrm{X}$ & $X$ & MPI-ESM-MR & $X$ & & $\mathrm{X}$ \\
\hline FIO-ESM & $\mathrm{X}$ & & $\mathrm{X}$ & MRI-CGCM3 & $\mathrm{X}$ & $X$ & $\mathrm{X}$ \\
\hline GFDL-CM3 & $\mathrm{X}$ & $\mathrm{X}$ & $\mathrm{X}$ & MRI-ESM1 & $\mathrm{X}$ & & \\
\hline GFDL-ESM2G & $\mathrm{X}$ & & $\mathrm{X}$ & NorESM1-M & $X$ & $\mathrm{X}$ & $\mathrm{X}$ \\
\hline GFDL-ESM2M & $X$ & X & $X$ & NorESM1-ME & $X$ & & X \\
\hline GISS-E2-H & $X$ & $X$ & $X^{*}$ & & & & \\
\hline
\end{tabular}

CMIP5 simulations used for the attribution analysis. We distinguish simulations with combined historical and RCP8.5 radiative forcing (hist) from 1902 to 2014 , simulations with only historical natural forcing (histNat) from 1902 to 2005, and long-term simulations with pre-industrial radiative forcing (pi). One initial condition simulation per model is considered, that is the "rli1p1" member. ${ }^{\star}$ Corresponds to member "r1i1p2" because no "r1i1p1" simulation is available. 
Extended Data Table 2 | Attribution of changes in dry season water availability to human-induced climate change based on reconstructions from individual land surface models

\begin{tabular}{ccccccccccc}
\hline & CESM2 & $\begin{array}{c}\text { CMCC- } \\
\text { ESM2 }\end{array}$ & $\begin{array}{c}\text { CNRM- } \\
\text { CM6-1 }\end{array}$ & $\begin{array}{c}\text { CNRM- } \\
\text { ESM2-1 }\end{array}$ & E3SM & $\begin{array}{c}\text { IPSL- } \\
\text { CM6A-LR }\end{array}$ & $\begin{array}{c}\text { CNRM-CM6- } \\
\text { 1-cruNcep }\end{array}$ & $\begin{array}{c}\text { LSM } \\
\text { DDM }\end{array}$ \\
\hline Hist. & 0.92 & 0.78 & 1 & 1 & 0.69 & 1 & 0.92 & 0.99 & 1 \\
Hist. Nat. & 0.66 & 0.08 & 0.84 & 0.73 & 0.28 & 0.37 & 0.42 & 0.56 & 0.36 \\
\hline
\end{tabular}

Analysis analogous as for Fig. 2 from the article. Values indicate the probability that the spatial pattern Spearman correlation between multi-model mean $\Delta(P-E T)$ from historical simulations and from a single land surface model reconstruction is greater than what is expected from natural climate variability. Results are shown for historical simulations with human-induced plus natural radiative forcing (Hist) and for simulations with natural radiative forcing only (Hist. Nat.). The probabilities for the multi-model mean of the land surface model reconstructions with GSWP-3 forcing (LSM) as well as for the data-driven reconstruction (DDM) are also provided. 